\title{
Tracking Arteriovenous Fistula Maturation: A Novel Approach
}

\author{
Laura M. Rosales ${ }^{\mathrm{a}}$ Hanjie Zhang ${ }^{\mathrm{a}}$ Marilou Mateo $^{\mathrm{a}}$ Brenda Chan $^{\mathrm{a}}$ \\ Israel Campos ${ }^{c}$ Nikolas Harbord $^{\text {b,d }}$ Stephan Thijssen ${ }^{a}$ Peter Kotanko ${ }^{a, b}$ \\ ${ }^{a}$ Renal Research Institute, New York, NY, USA; ${ }^{b}$ Icahn School of Medicine at Mount Sinai, New York, NY, USA; \\ 'Department of Educational and Research, NausLife Hemodialysis Clinics, Morelia, Mexico; ${ }^{\mathrm{d}}$ Mount Sinai Beth Israel \\ Medical Center, New York, NY, USA
}

\section{Keywords}

Arteriovenous fistula · Maturation · Central-venous oxygen saturation · Upper body blood flow · Vascular access

\begin{abstract}
Background: The time between the creation of an arteriovenous fistula (AVF) and its successful use is significantly longer in hemodialysis (HD) patients in the United States compared to those in other countries, and there is an urgent need to reduce the residence time of central-venous catheters (CVC). Methods: Successful AVF creation and maturation results in typical hemodynamic changes, such as an increase in cardiac output and upper body blood flow (UBBF). In patients with CVC as vascular access, we measured once per minute intradialytic central-venous oxygen saturation $\left(\mathrm{ScvO}_{2}\right)$ and hemoglobin levels simultaneously using the Crit-Line Monitor. Under conditions of stable upper body oxygen consumption and arterial oxygen saturation, $\mathrm{ScvO}_{2}$ and hemoglobin concentration allows the calculation of estimated UBBF (eUBBF). In a quality improvement project, we used $\mathrm{ScvO}_{2}$ and eUBBF to track the hemodynamic changes accompanying AVF maturation. Results: Out of 11 patients ( 9 incident to HD, 1 female, age $61 \pm 13$ years), AVF maturation was successful in 9 .
\end{abstract}

In 1 patient, the AVF did not mature. One patient died from sudden cardiac death with a maturing AVF. In the 9 patients with successful AVF maturation, $\mathrm{ScVO}_{2}$ increased from $60.9 \pm$ 2.7\% prior to AVF creation to $73.4 \pm 3.6 \%$ a week after AVF creation (19.6 $\pm 6.3 \%$ increase). eUBBF increased from $1.3 \pm$ 0.3 to $2.2 \pm 0.6 \mathrm{~L} / \mathrm{min}(62.7 \pm 37.5 \%$ increase); no material $\mathrm{ScvO}_{2}$ or eUBBF changes occurred in the other 2 patients. Conclusion: Our results indicate the potential utility of $\mathrm{ScvO}_{2}$ and eUBBF to track the hemodynamic response to AVF maturation. To what extent these insights translate into shortening of the time between AVF creation and successful cannulation warrants further investigations.

๑) 2018 S. Karger AG, Basel

\section{Background}

The creation of a native arteriovenous fistula (AVF) as a primary vascular access over AV grafts and centralvenous catheters (CVC) in hemodialysis (HD) patients has been recommended for decades by the National Kidney Foundation - Kidney Disease Outcomes Quality Initiative and other group initiatives. An AVF is favored due to its advantage over CVC usage with respect to sur-

\section{KARGER}

(c) 2018 S. Karger AG, Basel

E-Mail karger@karger.com

www.karger.com/bpu
Laura M. Rosales, MD

Renal Research Institute

315 East, 62 Street, 4 floor

New York, NY 10065 (USA)

E-Mail laura.rosales@ rriny.com 
vival outcomes, hospitalization rates, and cost to the healthcare system [1-4]. Notwithstanding this evidence, most US incident patients start HD using a CVC [5-8]. Even though the use of AVF has notably improved in prevalent patients, the initiation of $\mathrm{HD}$ with an AVF continues to be low. In the United States, about $80 \%$ of patients initiate HD with a CVC as vascular access [911 .

The time between AVF creation and first use depends on a successful AVF maturation, which varies significantly within and between countries $[12,13]$. The Kidney Disease Outcomes Quality Initiative proposed opinionbased ultrasound criteria for successful AVF maturation of $600 \mathrm{~mL} / \mathrm{min}$ blood flow rate, $0.6 \mathrm{~cm}$ diameter, and $\leq 0.6$ $\mathrm{cm}$ depth from the skin ("rule of sixes") [14], or empirically, as the ability to cannulate the AVF with 2 needles and obtain sufficient blood flow for adequate $\mathrm{HD}$ for at least 30 consecutive days [14-17]. Once an AVF has been cannulated successfully with 2 needles, the CVC can be removed. HD patients with a newly created AVF may continue to use CVCs for prolonged periods because of inappropriate maturation or failure to maintain patency. The extended use of a CVC is associated with adverse outcomes $[12,18-20]$.

The time to first AVF cannulation differs between countries and is longer in the United States than that in most other countries $[1,13]$. Routine assessment of AVF maturation is largely subjective, based on clinical assessment performed by the nursing staff, whose experience and standards of practice may vary from clinic to clinic. A recent multicenter, observational cohort study in 602 participants identified AFV blood flow rate, diameter, and depth as moderate predictors of AVF maturation [21]. However, there are no generally agreed upon hemodynamic criteria for determining AVF maturity and cardiovascular adaptation to the AVF [22]. Additionally, there is no agreed upon process to follow fistula maturation and attempt cannulation based on prior hemodynamic information. Moreover, the approach to a timely fistula maturation and successful cannulation may be performed unreliably at some clinics [23].

Clinical studies have shown that cardiac output and upper body blood flow (UBBF) increase following AVF creation. This increased perfusion of the upper body can be expected to result in higher central-venous oxygen saturation $\left(\mathrm{ScvO}_{2}\right)$. Motivated by that physiological reasoning we embarked on a quality improvement program (QIP) to reduce CVC residence time by following AVF maturation using $\mathrm{ScvO}_{2}$ and calculation of estimated UBBF (eUBBF).

AVF Maturation Tracking

\section{Methods}

This QIP took place at 2 Renal Research Institute clinics in New York City. The participation included incident and prevalent HD patients with a CVC as vascular access, with a newly created AVF between April 2017 and September 2018. In the short term, we considered AVF maturation as the time between AVF creation and first use, when the AVF allowed cannulation by 2 large bore needles, achieving sufficient blood flows for an adequate $\mathrm{HD}$, and CVC removal was prospectively scheduled. The clinics use the Crit-Line Monitor (Fresenius Medical Care, Waltham, MA, USA) during HD as part of the standard of care. The Crit-Line Monitor is approved by the US Food and Drug Administration for measurement of hematocrits and oxygen saturation. All patients received bicarbonate dialysis and used polysulfone membranes.

The Crit-Line Monitor automatically and noninvasively measures oxygen saturation and hematocrit in the extracorporeal circuit during HD 9,000 times per minute and reports their respective average once per minute. Per manufacturer, the Crit-Line's accuracy for oxygen saturation measurement is within $2 \%$.

For estimation of upper body oxygen consumption we considered the brain and upper body skeletal muscle tissue, with the brain being by far the greatest consumer of oxygen at rest. Therefore, the upper body oxygen consumption was calculated as the sum of oxygen consumption of the brain and muscle. The respective brain tissue masses were calculated using anthropometric formulae [24]. Muscle mass was estimated as $2.3 \mathrm{~kg}$ in males and $1.2 \mathrm{~kg}$ in females. We then multiplied the estimated organ mass with organ-specific oxygen uptake rates, 3.5 of $\mathrm{O}_{2} / 100 \mathrm{~g}$ brain tissue/min and $0.2 \mathrm{~mL}$ of $\mathrm{O}_{2} / 100 \mathrm{~g}$ resting muscle tissue/min, respectively.

Ignoring the negligible amount of dissolved oxygen in the blood, the arterial oxygen content in $\mathrm{mL}$ of $\mathrm{O}_{2}$ per liter of blood is defined as: (equation [1])

$$
\mathrm{CaO}_{2}=1.34 \times \mathrm{Hgb} \times \mathrm{SaO}_{2}
$$

with 1.34 being the $\mathrm{mL}$ of $\mathrm{O}_{2}$ that $1 \mathrm{~g}$ of $\mathrm{Hgb}$ can bind if fully saturated, Hgb being the hemoglobin concentration in $\mathrm{g} / \mathrm{L}$, and $\mathrm{SaO}_{2}$ being the arterial blood oxygen saturation expressed as fraction.

Analogously, central-venous blood oxygen content in $\mathrm{mL}$ of $\mathrm{O}_{2}$ per liter of blood is defined as: (equation [2])

$$
\mathrm{CcvO}_{2}=1.34 \times \mathrm{Hgb} \times \mathrm{ScvO}_{2}
$$

The rate of oxygen delivery to the upper body is the product of arterial oxygen content and UBBF rate. Similarly, if a CVC resides in the superior vena cava, the rate of oxygen return from the upper body is the product of central-venous blood oxygen content and UBBF. The difference between the two equals the rate of oxygen consumption ( $\mathrm{utilO}_{2}$ ) by the tissues supplied by the UBBF. This is illustrated in the following mass balance equation: (equation [3]).

$$
\mathrm{UBBF} \times \mathrm{CaO}_{2}-\mathrm{utilO}_{2}=\mathrm{UBBF} \times \mathrm{CcvO}_{2}
$$

with UBBF being the upper-body blood flow rate in $\mathrm{L} / \mathrm{min}$, $\mathrm{utilO}_{2}$ being the rate of oxygen consumption in $\mathrm{mL} / \mathrm{min}$ by the tissues supplied by the UBBF. Rearranging equation (3) to: 
Fig. 1. Schematic representation of arteriovenous fistula (AVF) maturation tracking using central-venous oxygen saturation $\left(\mathrm{ScvO}_{2}\right)$. The green line represents adequate AVF maturation and adequate hemodynamic adaptation. Red lines depict inadequate AVF maturation and/or inadequate hemodynamic adaptation.

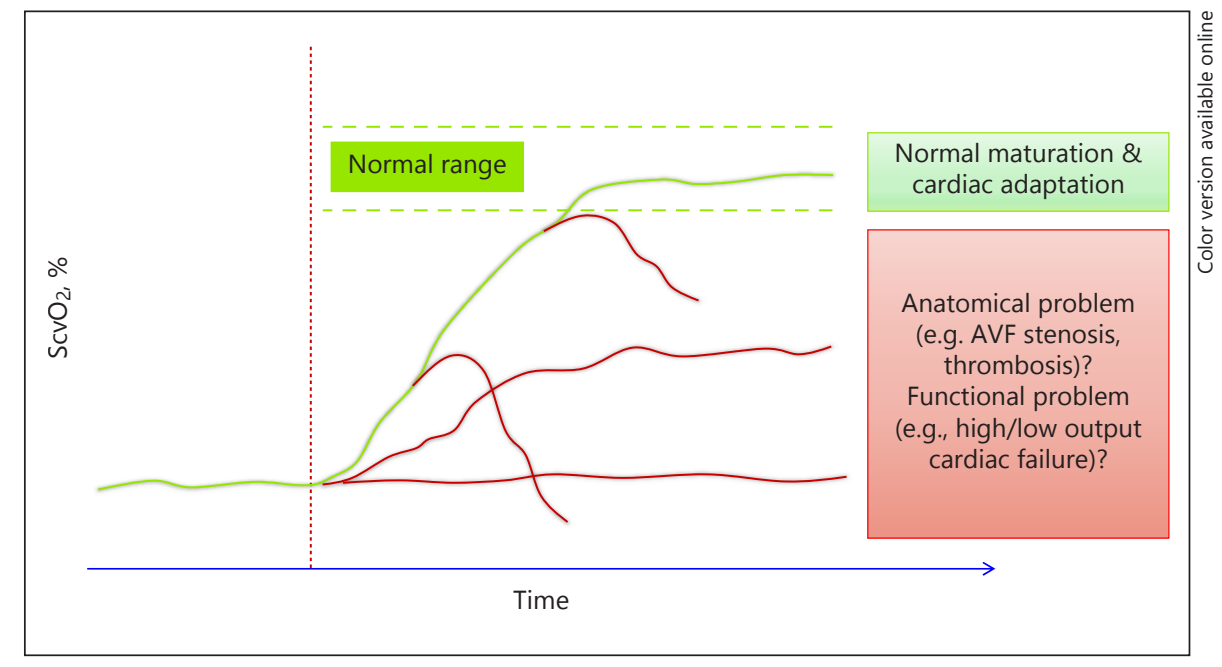

$$
\mathrm{UBBF}=\frac{\mathrm{utilO}_{2}}{\mathrm{CaO}_{2}-\mathrm{CcvO}_{2}}
$$
[4])

Substituting equations (1) and (2) from above yields: (equation

$$
\mathrm{UBBF}=\frac{\mathrm{utilO}_{2}}{1.34 \times \mathrm{Hgb} \times\left(\mathrm{SaO}_{2}-\mathrm{ScvO}_{2}\right)}
$$

To illustrate the relationship between $\mathrm{ScvO}_{2}$ and the other parameters, the equation can be further rearranged as follows: (equation [5])

$$
\mathrm{ScvO}_{2}=\mathrm{SaO}_{2}-\frac{\mathrm{utilO}_{2}}{1.34 \times \mathrm{Hgb} \times \mathrm{UBBF}}
$$

The UBBF equation above illustrates that in order to calculate $\mathrm{UBFB}$, the following parameters are required: the rate of oxygen consumption by the tissues supplied by the UBBF, the Hgb concentration, $\mathrm{SaO}_{2}$ and $\mathrm{ScvO}_{2}$.

Arterial and central-venous $\mathrm{Hgb}$ concentration can be considered identical. In our study, we obtained $\mathrm{ScvO}_{2}$ and simultaneous $\mathrm{Hgb}$ noninvasively using the Crit-Line Monitor. This input allows us together with reasonable assumptions regarding oxygen consumption and $\mathrm{SaO}_{2}$ to calculate an estimated UBBF (eUBBF). To mitigate the impact of ultrafiltration on $\mathrm{ScvO}_{2}$ and $\mathrm{Hgb}$, we used data collected only between min 5 and 20 into the treatment.

Patients were followed with the Crit-Line Monitor while receiving HD through time periods before and after AVF creation. With normal AVF maturation and hemodynamic adaptation, the $\mathrm{ScvO}_{2}$ and eUBBF are expected to increase. In contrast, AVF maturation failure and insufficient hemodynamic adaptation are expected to be blunted or absent from $\mathrm{ScvO}_{2}$ and eUBBF increases (Fig. 1). It is important to point out that failure to increase $\mathrm{ScvO}_{2}$ and eUBBF may indicate either failed AVF maturation or inadequate hemodynamic adaptation. Discriminating these pathologies requires additional investigations.

We report data as mean \pm SD or percent. Because of the small number of patients, we refrained from formal statistical testing.

\section{Results}

We started the QIP in April 2017. There were 2 prevalent and 9 incident HD patients who underwent AVF creation. Their age was $61 \pm 13$ years, 10 males, 6 Whites, 4 African Americans, and 1 Asian. The HD vintage was $3.5 \pm 7$ years (range $0.1-17$ ). The AVF location was brachiocephalic in 9 and radiocephalic in 2 patients.

Out of these 11 patients, AVF maturation was successful in 9. In these 9 patients, $\mathrm{ScvO}_{2}$ increased from $60.9 \pm$ $2.7 \%$ prior to $\mathrm{AVF}$ creation to $73.4 \pm 3.6 \%$ a week later (increase by $19.6 \pm 6.3 \%$; Fig. 2); eUBBF increased from $1.3 \pm 0.3$ to $2.2 \pm 0.6 \mathrm{~L} / \mathrm{min}$ (increase by $62.7 \pm 37.5 \%$; Fig. 3). We saw no material $\mathrm{ScvO}_{2}$ or eUBBF changes in the 2 patients who suffered from complications. One patient died from sudden cardiac death with a patent AVF in place. In the other patient the AVF failed to mature and assisted maturation procedures were required due to small and noncompliant vessels.

\section{Discussion}

The main finding of this pilot QIP is an increase of $\mathrm{ScvO}_{2}$ and eUBBF in patients with uncomplicated AVF maturation; in contrast, these parameters remained essentially unchanged in patients who experienced complications (primary AVF maturation failure in 1 patient and presumably cardiac maladaptation after AVF placement in the other). Monitoring the dynamics of $\mathrm{ScvO}_{2}$ and $\mathrm{eU}$ BBF may allow the early detection of insufficient AVF maturation and/or poor hemodynamic adaptation, whereas observing their expected increase may aid the 


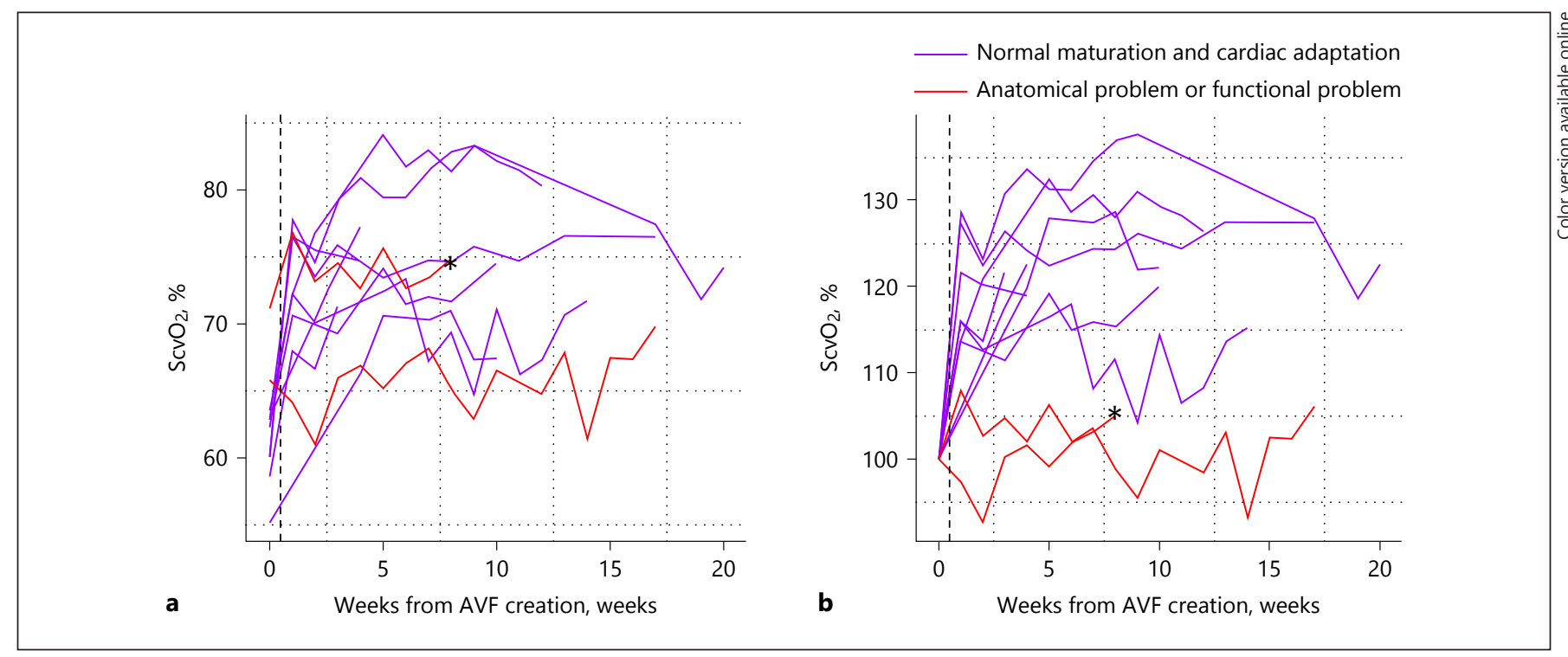

Fig. 2. a Time course of absolute $\mathrm{ScvO}_{2}$ dynamics in 9 patients with normal AVF maturation (purple lines) and 2 patients with inadequate hemodynamic adaptation and failed AVF maturation respectively (red lines). b Time course of $\mathrm{ScvO}_{2}(\%)$ changes relative to levels prior to AVF creation. The lines end at first successful AVF use (purple lines) or end of observation (red lines). The * denotes the deceased patient.

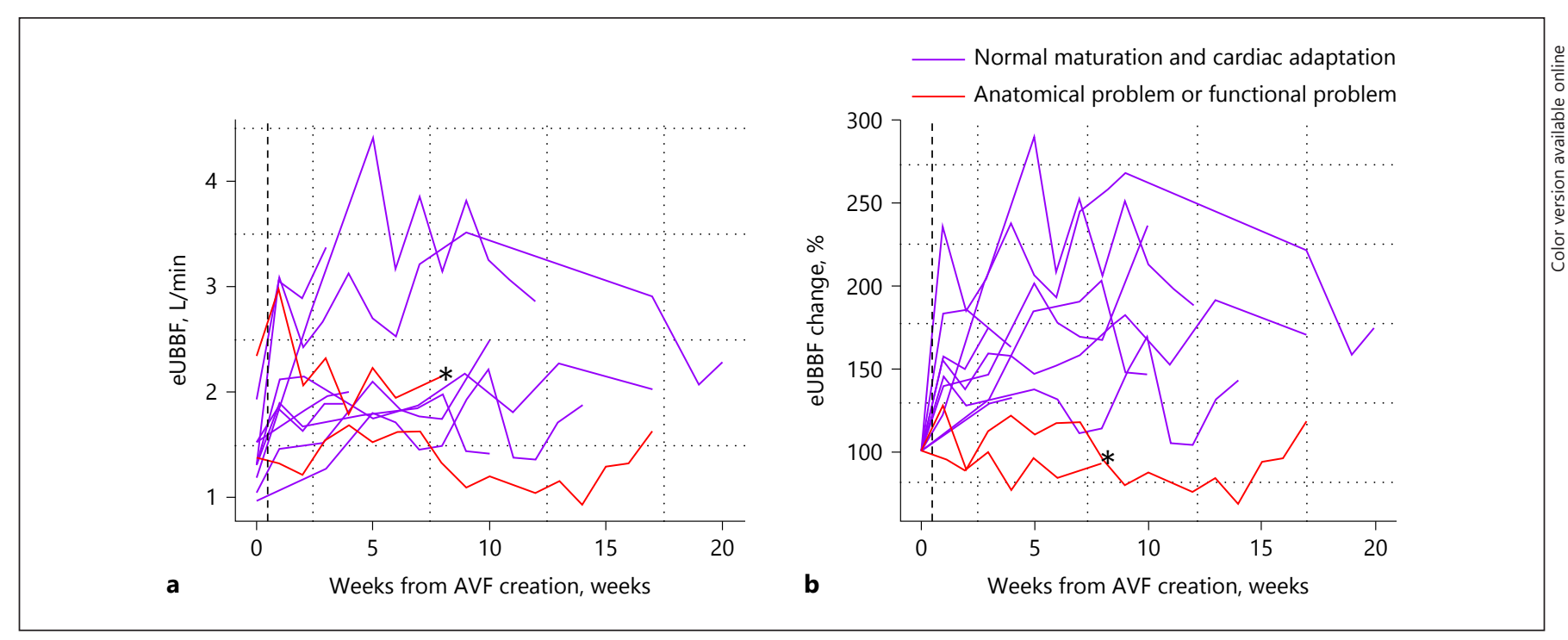

Fig. 3. a Time course of absolute eUBBF dynamics in 9 patients with normal AVF maturation (purple lines) and 2 patients with inadequate hemodynamic adaptation and failed AVF maturation respectively (red lines). b Time course of eUBBF changes relative

decision to cannulate, which may serve to reduce CVC residence times.

There is unambiguous evidence from numerous studies that CVC use is associated with increased morbidity and mortality in maintenance HD patients. One obvious to levels prior to AVF creation. The lines end at first successful AVF use (purple lines) or end of observation (red lines). The * denotes the deceased patient.

way to reduce CVC use is to shorten the time between AVF creation and cannulation. In 2004, a DOPPS study indicated substantial differences in time to AVF cannulation between countries [25]. The fraction of patients having their AVF cannulated in the first month after creation 
was $74 \%$ in Japan, $50 \%$ in Europe, and 2\% in the United States. The respective numbers after 2 months were $98 \%$ (Japan), 79\% (Europe), and 36\% (US). In a recent study from the United States, Woodside et al. [5] used CrownWeb data to characterize time to the first use of newly created AVF as surrogate for successful AVF maturation. The authors identified 45,087 new AVFs that were placed in 39,820 prevalent HD patients in 2013. No evidence of AVF use was found in $36.2 \%$ of AVFs until December 31, 2014. Only $17.1 \%$ were cannulated by 2 months and $54.7 \%$ within 4 months. A time to first cannulation $>10$ months was reported in $6.6 \%$.

Against this background, we explored the use of pointof-care measurement of $\mathrm{ScvO}_{2}$ to provide caregivers with an actionable bio-signal that reflects the combined effects of AVF maturation and hemodynamic adaptation. The physiological reasoning underlying that approach is straight forward. A successful AVF maturation results in a drop in upper body peripheral vascular resistance and hence blood pressure, resulting in baroreceptor activation and subsequent hemodynamic adaptations, including a rise in cardiac output [26] and UBBF. While the relative distribution between UBBF and lower body blood flow in that situation has, to the best of our knowledge, not been studied systematically, it is reasonable to assume a directional agreement between UBBF and cardiac output. A higher UBBF results in an increased oxygen delivery to the upper body. Under conditions of constant tissue oxygen uptake, hemoglobin concentration and $\mathrm{SaO}_{2}$, the $\mathrm{ScvO}_{2}$ can be expected to increase, as shown in equation (5). For tracking UBBF from treatment to treatment, we must further assume that the oxygen consumption rate by the upper-body tissues remains constant over the period during which UBBF is assessed.

While it is difficult to assess upper body $\mathrm{O}_{2}$ uptake in a patient on a routine clinical basis, it is important that no major changes in a stable patient at rest should be expected within a few weeks. While HD patients may have a lower brain oxygen consumption compared to healthy subjects [27], the assumption of a stable brain oxygen uptake in a given patient is reasonable, unless the mass of viable brain tissue changes substantially. The same applies to resting muscle oxygen uptake. In a clinically stable patient, changes in body composition take place on a time scale of months rather than days or weeks (the time expected for AVF maturation). In order to quantitate brain oxygen uptake, we estimated the respective brain mass using anthropometric formulae [24]. Factors that can change within minutes and thus affect $\mathrm{ScvO}_{2}$ are $\mathrm{SaO}_{2}$ and hemoglobin levels, the latter particularly during ultrafiltration. The Crit-Line Monitor simultaneously measures the oxygen saturation and hemoglobin concentration, so that the dynamic hemoglobin changes can be factored into the calculation. Concurrent measurements of $\mathrm{SaO}_{2}$ would be highly desirable because $\mathrm{SaO}_{2}$ can change throughout HD [28, 29]. We recognize the absence of $\mathrm{SaO}_{2}$ measurements as a limitation, and efforts to remedy this are underway. Arterial and central-venous Hgb concentration can be considered identical. In our study, we obtained $\mathrm{ScvO}_{2}$ and $\mathrm{Hgb}$ noninvasively using the Crit-Line Monitor. In the absence of $\mathrm{SaO}_{2}$ measurements, we assumed a constant value of $92.6 \%$ for every patient, which was recently described to be the average $\mathrm{SaO} 2$ in a large population of $\mathrm{HD}$ patients [28]. It is important to appreciate that relative changes in $\mathrm{ScvO}_{2}$ and eUBBF appear to be more informative than their absolute values, mitigating concerns that the calculation of eUBBF is based on patient-specific assumptions that may be difficult to ascertain in clinical practice.

It is interesting to observe that substantial changes in $\mathrm{ScvO}_{2}$ and eUBBF are already present in the first week after AVF creation. These findings are in line with the rapid increase of AVF blood flow observed in ultrasound studies in patients following AVF creation [21]. In this study, the median (interquartile range) AVF flow increased in the full cohort from $604(380-936 ; n=539$ patients) on day 1 after AVF creation to 801 (518-902; $n=$ 527) $\mathrm{mL} / \mathrm{min}$ in week 2 . This $~ 30 \%$ increase in AVF blood flow within 2 weeks is less that the $\sim 60 \%$ eUBBF increase we have observed between the period before AVF creation and the week thereafter, indicating that eU$\mathrm{BBF}$ may be a more pronounced signal.

The use of $\mathrm{ScvO}_{2}$ and eUBBF monitoring to track AVF maturation and hemodynamic adaptation is also operationally attractive, since it can be automated and scaled to a large number of HD patients. Unlike ultrasound studies, this method is operator-independent and requires no additional personnel.

In summary, our findings indicate that normal AVF maturation is accompanied by an increase in $\mathrm{ScvO}_{2}$ and eUBBF. Both $\mathrm{ScvO}_{2}$ and $\mathrm{Hgb}$ are indispensable for eU$\mathrm{BBF}$ computation, and the advent of the Crit-Line Monitor has made routine, noninvasive and quasi-continuous measurements of these 2 parameters during HD a reality. Future detailed physiological studies including hemodynamic monitoring [30] and AVF flow measurement are required to better understand the maturation dynamics and the associated hemodynamic adaptation. Efforts should be made to characterize the expected trajectories 
of $\mathrm{ScvO}_{2}$ and eUBBF during adequate AVF maturation and cardiac adaptation. Application of the proposed method in a larger population is required to test the hypothesis that the CVC residence times can be reduced by a clinically relevant degree.

\section{Acknowledgments}

P.K. holds stock in Fresenius Medical Care. S.T. holds performance shares in Fresenius Medical Care. The Renal Research Institute is a wholly owned subsidiary of Fresenius Medical Care.

\section{References}

1 Rayner HC, et al: Vascular access results from the Dialysis Outcomes and Practice Patterns Study (DOPPS): performance against Kidney Disease Outcomes Quality Initiative (K/ DOQI) Clinical Practice Guidelines. Am J Kidney Dis 2004;44(5 suppl 2):22-26.

2 Lok CE: Fistula first initiative: advantages and pitfalls. Clin J Am Soc Nephrol 2007;2:10431053.

3 Allon M: Current management of vascular access. Clin J Am Soc Nephrol 2007;2:786-800.

4 Vassalotti JA, et al: Fistula first breakthrough initiative: targeting catheter last in fistula first. Semin Dial 2012;25:303-310.

5 Woodside KJ, et al: Arteriovenous fistula maturation in prevalent hemodialysis patients in the United States: a national study. Am J Kidney Dis 2018;71:793-801.

6 Ravani P, et al: Associations between hemodialysis access type and clinical outcomes: a systematic review. J Am Soc Nephrol 2013;24: 465-473.

7 Solid CA, Carlin C: Timing of arteriovenous fistula placement and Medicare costs during dialysis initiation. Am J Nephrol 2012;35: 498-508.

8 Pisoni RL, et al: Trends in US vascular access use, patient preferences, and related practices: an update From the US DOPPS practice monitor with international comparisons. Am J Kidney Dis 2015;65:905-915.

9 Malas MB, et al: Trends in incident hemodialysis access and mortality. JAMA Surg 2015; 150:441-448.

10 Collins AJ, et al: The state of chronic kidney disease, ESRD, and morbidity and mortality in the first year of dialysis. Clin J Am Soc Nephrol 2009;4(suppl 1):S5-S11.
11 Arhuidese IJ, et al: Utilization, patency, and complications associated with vascular access for hemodialysis in the United States. J Vasc Surg 2018;68:1166-1174.

12 Viecelli AK, et al: Vascular access outcomes reported in maintenance hemodialysis trials: a systematic review. Am J Kidney Dis 2018;71: 382-391.

13 Pisoni RL, et al: International differences in the location and use of arteriovenous accesses created for hemodialysis: results from the dialysis outcomes and practice patterns study (DOPPS). Am J Kidney Dis 2018;71:469-478.

14 NKF-KDOQI, Clinical Practice Guidelines and Recommendations for Vascular Access, 2006.

15 Hentschel DM: Determinants of arteriovenous fistula maturation. Clin J Am Soc Nephrol 2018;13:1307-1308.

16 Allon M, et al: Relationships between clinical processes and arteriovenous fistula cannulation and maturation: a multicenter prospective cohort study. Am J Kidney Dis 2018;71: 677-689.

17 Sequeira A, Naljayan M, Vachharajani TJ: Vascular access guidelines: summary, rationale, and controversies. Tech Vasc Interv Radiol 2017;20:2-8

18 USRDS, 2016 USRDS Annual Data Report on ESRD. Am J Kidney Dis 2017;69(3 supplement 1):A1-A8, S1-S688 (March 2017).

19 Taylor G, et al: Incidence of bloodstream infection in multicenter inception cohorts of hemodialysis patients. Am J Infect Control 2004;32:155-160.

20 Saran R, Pisoni RL, Weitzel WF: Epidemiology of vascular access for hemodialysis and related practice patterns. Contrib Nephrol 2004;142:14-28.
21 Robbin ML, et al: Prediction of arteriovenous fistula clinical maturation from postoperative ultrasound measurements: findings from the hemodialysis fistula maturation study. J Am Soc Nephrol 2018;pii:ASN.2017111225.

22 Basile C. Lomonte C: The complex relationship among arteriovenous access, heart, and circulation. Semin Dial 2018;31:15-20.

23 Segal M, Qaja E: Fistula types; in StatPearls. Treasure Island (FL), 2018.

24 Mehrpour O, et al: Brain weight of Iranian population; the first report. J Forensic Leg Med 2010;17:426-431.

25 Saran R, et al: Timing of first cannulation and vascular access failure in haemodialysis: an analysis of practice patterns at dialysis facilities in the DOPPS. Nephrol Dial Transplant 2004;19:2334-2340.

26 Basile C, et al: The relationship between the flow of arteriovenous fistula and cardiac output in haemodialysis patients. Nephrol Dial Transplant 2008;23:282-287.

27 Kanai H, et al: Depressed cerebral oxygen metabolism in patients with chronic renal failure: a positron emission tomography study. Am J Kidney Dis 2001;38(4 suppl 1):S129S133.

28 Meyring-Wosten A, et al: Intradialytic hypoxemia and clinical outcomes in patients on hemodialysis. Clin J Am Soc Nephrol 2016;11: 616-625.

29 Campos I, et al: Intradialytic hypoxemia in chronic hemodialysis patients. Blood Purif 2016;41:177-187.

30 Kitzler TM, et al: Noninvasive measurement of cardiac output in hemodialysis patients by task force monitor: a comparison with the Transonic System. ASAIO J 2007;53:561-565. 